\title{
Study of muons from ultra-high energy cosmic ray air showers measured with the Telescope Array experiment
}

\author{
R. Takeishi* for the Telescope Array Collaboration \\ Institute for Cosmic Ray Research, University of Tokyo, Kashiwa, Chiba, Japan \\ E-mail: take@icrr.u-tokyo.ac.jp
}

\begin{abstract}
The origin of ultra high energy cosmic rays (UHECR) is a long-standing mystery. The aim of the Telescope Array (TA) experiment is to reveal this by observing the spectrum, anisotropy, and mass composition by utilizing an array of surface detectors (SD) and fluorescence detectors. One of the uncertainties in UHECR observation derives from the hadronic interaction model used for air shower Monte Carlo (MC) simulations. The number of muons observed at the ground from UHECR induced air showers is expected to depend upon the composition of primary cosmic rays. The MC prediction depends also on hadronic interaction models. One may test the hadronic interaction models by comparing the measured number of muons with the $\mathrm{MC}$ prediction.

We studied muon densities in the UHE extensive air showers by analyzing the signal of surface detector stations for highly inclined showers which should have high muon purity. A high muon purity condition is imposed that requires the geometry of the shower and relative position of the given station. The condition implies that the muons dominate the signal, i.e. contribute about $\sim 65 \%$ of the total signal. The number of particles from air showers observed on the condition of muon purity is typically $1.88 \pm 0.08$ (stat.) \pm 0.43 (syst.) times larger than the MC prediction value using the QGSJET II-03 model for protons. The lateral distribution of the muons also falls more slowly in the data than the MC, causing larger discrepancy at larger lateral distance. The same feature was also obtained for other hadronic models, such as QGSJET II-04.
\end{abstract}

35th International Cosmic Ray Conference - ICRC2017

10-20 July, 2017

Bexco, Busan, Korea

${ }^{*}$ Speaker. 


\section{Introduction}

Cosmic rays with energies above about $10^{18} \mathrm{eV}$ are called ultra-high energy cosmic rays (UHECRs). The origin of UHECR is a long-standing mystery. The Telescope Array (TA) experiment [1], which is located in Utah, USA, aims to reveal the origin of UHECR by measuring the spectrum, the anisotropy and the mass composition of UHECR. When UHECRs enter the atmosphere, they interact with atmospheric nuclei and generate the particle cascade, which is called air shower. The information of primary cosmic rays is estimated from the observed signal of air shower particles and the air shower Monte Carlo (MC) simulation.

UHECR air showers are not fully understood, and the observation results of the mass composition have the dependence of hadronic models used in the air shower MC [2] 3]. Recently, the Pierre Auger Observatory, which is located in Mendoza, Argentina, reported [4] that the average ratio of the number of muons observed on the ground to the MC prediction is $1.841 \pm 0.029$ (stat.) \pm 0.324(syst.) using QGSJET II-03 model [5】 for proton. The number of muons in the Auger data is also larger than the MC prediction for iron. In addition to that, the Auger group reported that the observed hadronic signal in UHECR air showers is $1.61 \pm 0.21(1.33 \pm 0.16)$ times larger than the MC prediction values for QGSJET II-04 [6] (EPOS-LHC [7]) model, including statistical and systematic errors [8]. Thus, the Auger group claimed the excess of muons in the data.

The number of muons from the UHECR on the ground depends on the mass composition of primary cosmic rays. Its MC prediction depends also on hadronic interaction models. One may test the hadronic interaction models by comparing the measured number of muons with the MC prediction. The TA experiment uses the surface detector (SD) made of plastic scintillator [9]. It is sensitive to the electromagnetic (EM) component that is the major part of secondary particles from the air showers. An analysis approach to increase the muon purity is necessary to study the number of muons in the TA SD data. This work aims to develop an analysis of muons from the UHECR air showers by using TA SD and study the difference of the number of muons between the experimental data and the MC.

The analysis strategy is as follows:

1. Assume the cosmic ray mass composition is pure proton.

2. Define the condition of the high muon purity using the MC.

3. Compare the observed signal size from air shower particles with the MC prediction under the high muon purity condition.

\section{Method}

The TA experiment is designed to measure air shower secondary particles on the ground with the SD and fluorescence light induced from the air shower with the fluorescence detector (FD). The TA SD array consists of 507 scintillation counters, placed on a square grid with $1.2 \mathrm{~km}$ spacing, covering $700 \mathrm{~km}^{2}$. The three TA FDs are located around the SD array, looking towards the sky above the array.

We use the TA SD 7 years' dataset recorded from 11 May 2008 through 11 May 2015, and the events reconstructed by the same method as the TA spectrum analysis [10] with energy range 

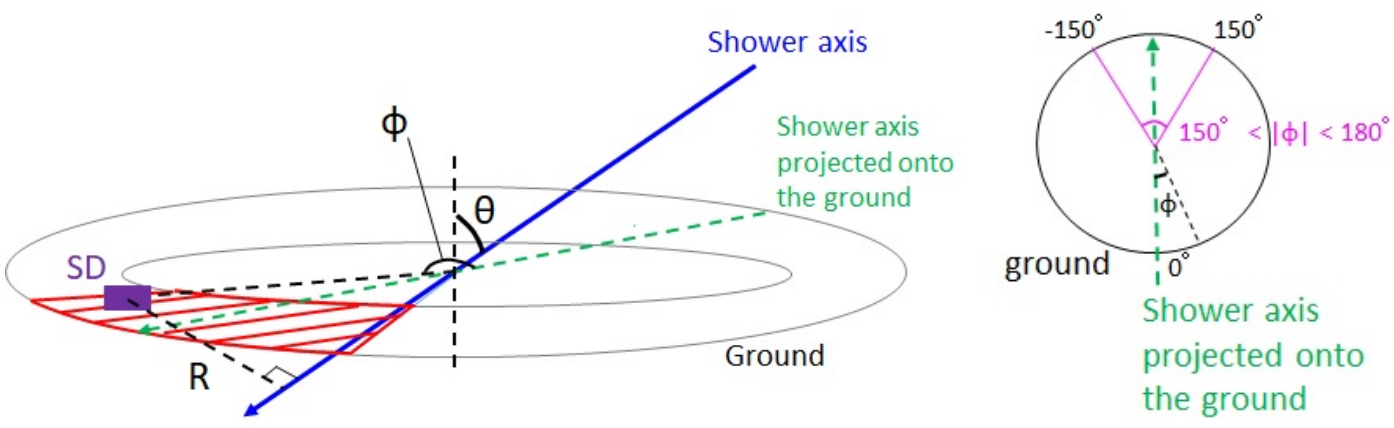

Figure 1: (left) Geometry definition of the muon analysis. SD location on the ground is selected by $\phi$ and $R$ to reduce EM background. The muon purity in the SD signal is calculated in each $(\phi, R)$ bin. The red region in the figure shows the bin where the distance from the particle generation points on the shower axis is relatively larger than other bins, which is expected to be the least EM background bin. (right) Top view for $\phi$ definition. There are six regions for the analysis and magenta lines show boundaries of $150^{\circ}<|\phi|<180^{\circ}$.

$10^{18.8} \mathrm{eV}<E<10^{19.2} \mathrm{eV}$. In the energy range, the mass composition of the primary cosmic rays is consistent with proton within statistical and systematic errors by $X_{\max }$ measurement using the TA FD [2, 31 11, 12], thus we use the MC for proton primaries. The experimental data is compared with the MC using the hadronic models QGSJET II-03, QGSJET II-04, Epos 1.99 [13] and Sibyll 2.1 [14].

The EM components are composed of electron and gamma. They are attenuated faster than muons compared in the same pass length, because the EM components largely lose their energy by the pair production and bremsstrahlung in the shower propagation but muons can penetrate the atmosphere down to the ground before they decay. Hence the ratio of the number of air shower muons to that of all charged particles in the same SDs (hereafter this ratio is described as the muon purity) is expected to be larger as the SDs are more distant from secondary particle generation points on the shower axis. We divide the detector hit in the air shower events of the dataset using $\theta$ (the zenith angle), $\phi$ (the azimuth angle relative to the shower arrival direction projected onto the ground), and $R$ (the distance from shower axis). The geometry definition is described in figure 1 . When $\theta,|\phi|$ or $R$ values become large, the pass length of air shower particles increases, then the muon purity in the signal of SDs is expected to be high.

For each event, SDs which have air shower signals are picked up and the integrated FADC for each waveform is calculated. The value by the unit of VEM (Vertical Equivalent Muon) is filled in the histogram of corresponding $(\theta, \phi, R)$ bin. Figure 2 shows the sample waveforms and histograms in each particle type. One detector signal deserves one entry in the histogram. If there is a SD in a $(\phi, R)$ bin but it does not have any signal, then one entry is filled to the corresponding bin of the histogram as 0 VEM. Figure 3 shows the lateral distributions of the signal and the muon purity. For the high muon purity condition $\left(30^{\circ}<\theta<45^{\circ}, 150^{\circ}<|\phi|<180^{\circ}, 2000 \mathrm{~m}<R<4000 \mathrm{~m}\right)$, the muon purity is mainly $60-70 \%$, and we use this condition for the comparison of the data with the MC.

The systematic uncertainty in this study is mainly caused by the uncertainty of the TA FD energy measurement $21 \%$ [15]. It causes the uncertainty of the signal size of the data, which is about 

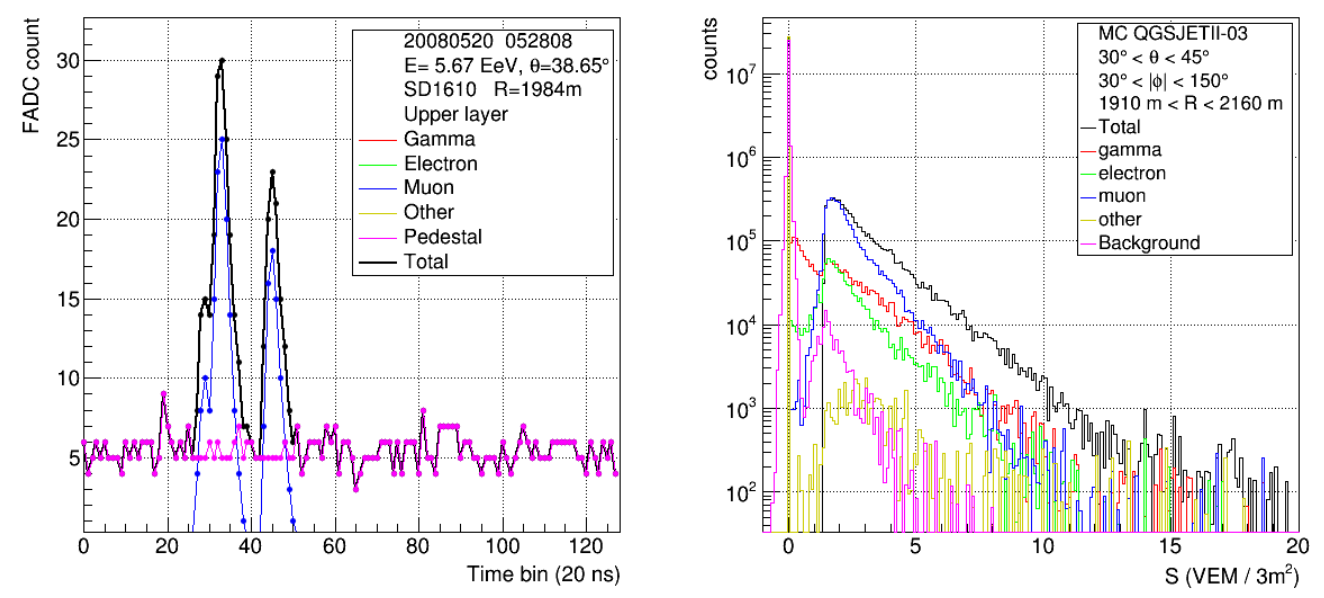

Figure 2: Sample event waveforms in a SD ( $3 \mathrm{~m}^{2}$ in area $)$ and the signal size distribution for different particle types by the MC. The red, green, blue, yellow, magenta and black represent gamma, electron, muon, other shower components, atmospheric muon background and the total of them, respectively. (left) Sample event waveforms in a SD for $E=5.67 \mathrm{EeV}, \theta=38.65^{\circ},|\phi|=175^{\circ}, R=1984 \mathrm{~m}$. (right) Histograms of the signal size (denoted as $S$ in the figure) in a SD for $10^{18.8} \mathrm{eV}<E<10^{19.2} \mathrm{eV}, 30^{\circ}<\theta<45^{\circ}, 150^{\circ}<|\phi|<180^{\circ}, 1910$ $\mathrm{m}<R<2160 \mathrm{~m}$.

21\%. Minor systematic uncertainties are from 1 MIP calibration (2\%), background atmospheric muon ( $0 \%-15 \%, R$ dependence), assumption of Poisson distribution for signal size histograms (5\% - 10\%, $R$ dependence), air shower reconstruction (5\% - 10\%, $R$ dependence) and SDs not working properly ( $\sim 1 \%, R$ dependence). The details of minor systematic uncertainties described above are under investigation.

\section{Results}

Figure 4 shows the lateral distributions of the signal and the ratio of the data to the MC for QGSJET II-03 model. The observed lateral distribution (black points) falls down slower than the MC. The typical ratios of the data to the MC for proton using QGSJET II-03 model are calculated to be $1.72 \pm 0.10$ (stat.) \pm 0.40 (syst.) at $1910 \mathrm{~m}<R<2160 \mathrm{~m}$ and $3.14 \pm 0.36$ (stat.) \pm 0.72 (syst.) at $2760 \mathrm{~m}<R<3120 \mathrm{~m}$.

Figure 5 shows the lateral distributions of the signal and the ratio of the data to the MCs with various hadronic interaction models; QGSJET II-03, QGSJET II-04, Epos 1.99 and Sibyll 2.1. The observed lateral distribution (black points) falls down slower than the MCs with all the hadronic interaction models (colors).

Cosmic rays which are heavier than iron are unfavorable if the source is an astrophysical object. We generated the lateral distributions assuming iron with QGSJET II-03 model by the $\mathrm{MC}$, and compared with that of the data. The left panel of figure 6 shows the lateral distributions of the ratio of the data to the MCs for proton and iron. The average signal of the data is larger than the MC for iron at $R \sim 3000 \mathrm{~m}$. For other $R$ conditions, they are within systematic errors. The right panel of figure 6 shows the correlation between the muon purity expected from the MC 

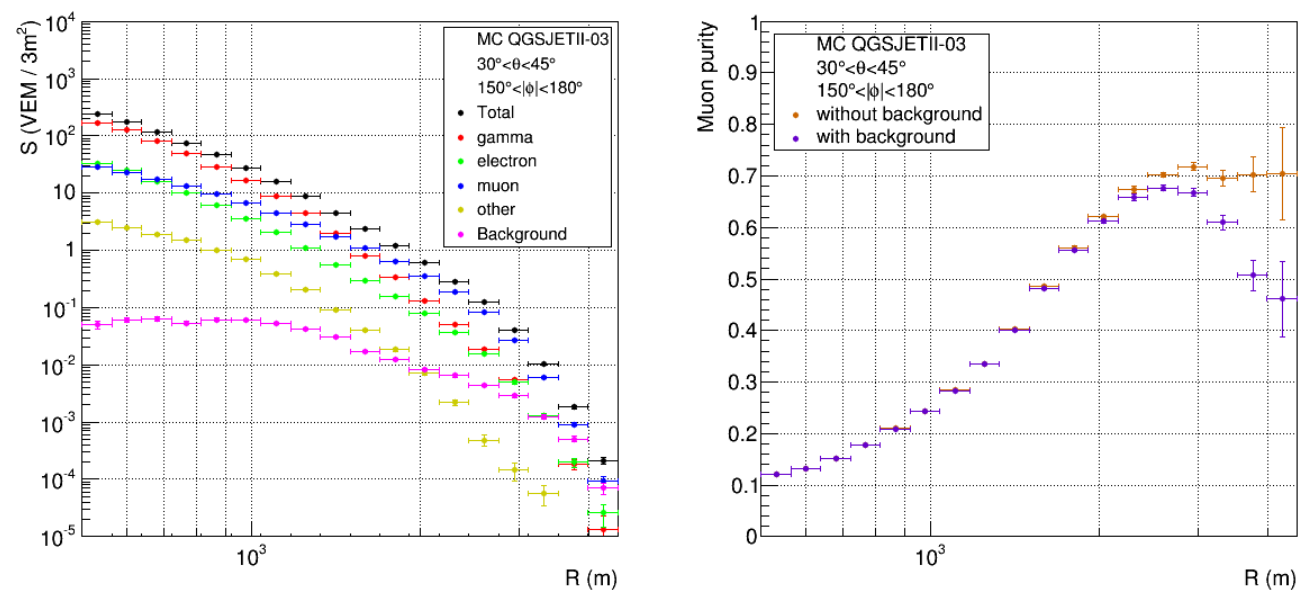

Figure 3: Lateral distributions of air showers of the MC with QGSJET II-03 model for $30^{\circ}<\theta<$ $45^{\circ}, 150^{\circ}<|\phi|<180^{\circ}, 500 \mathrm{~m}<R<4500 \mathrm{~m}$. The horizontal axis describes $R$, which is the distance from shower axis. The vertical error bar shows the standard deviation. (left) Lateral distributions of the average signal. The red, green, blue, yellow, magenta and black represent gamma, electron, muon, other shower components, atmospheric muon background and the total of them, respectively. (right) Lateral distributions of the muon purity. The violet and orange show the calculation with and without atmospheric muon background, respectively.
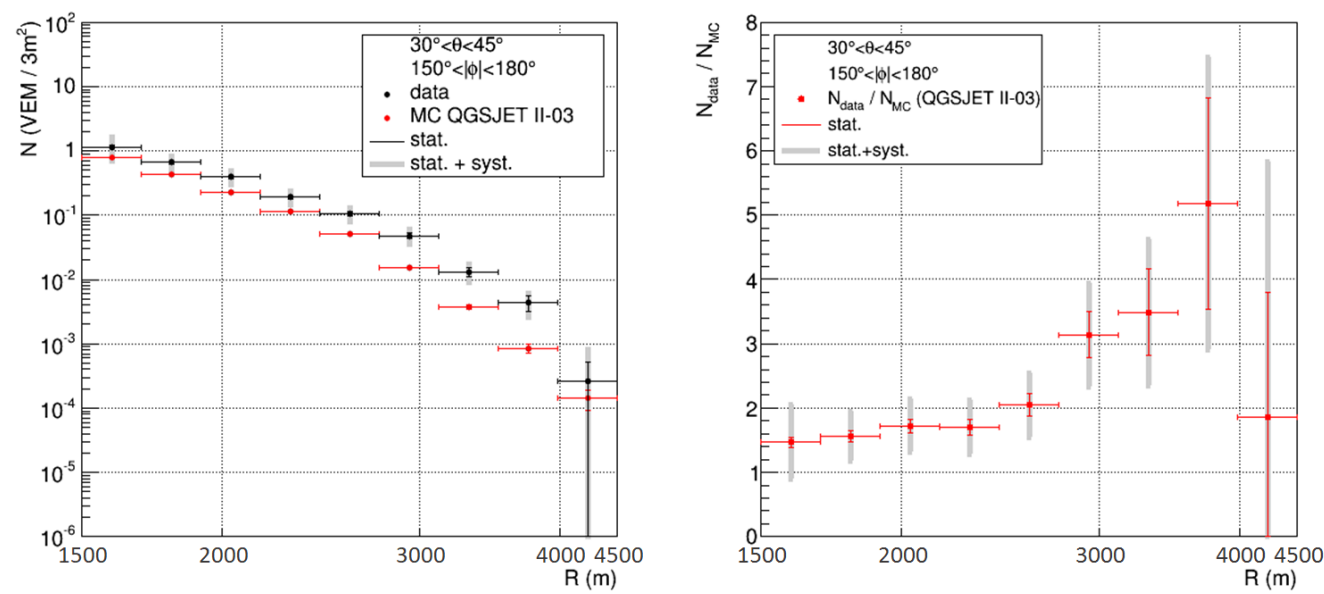

Figure 4: Lateral distributions of air showers of the data and the MC with various hadronic models for $30^{\circ}<\theta<45^{\circ}, 150^{\circ}<|\phi|<180^{\circ}, 1500 \mathrm{~m}<R<4500 \mathrm{~m}$. The vertical thin error bars and shaded grey thick error bars represent statistical error and quadratic sum of statistical and systematic errors, respectively. (left) Lateral distributions of the average signal assuming the histograms follow the Poisson distribution, denoted as $N$ in the figure. The black and red points represent the data and the MC, respectively. (right) The average ratio of the data to the $\mathrm{MC}$. 

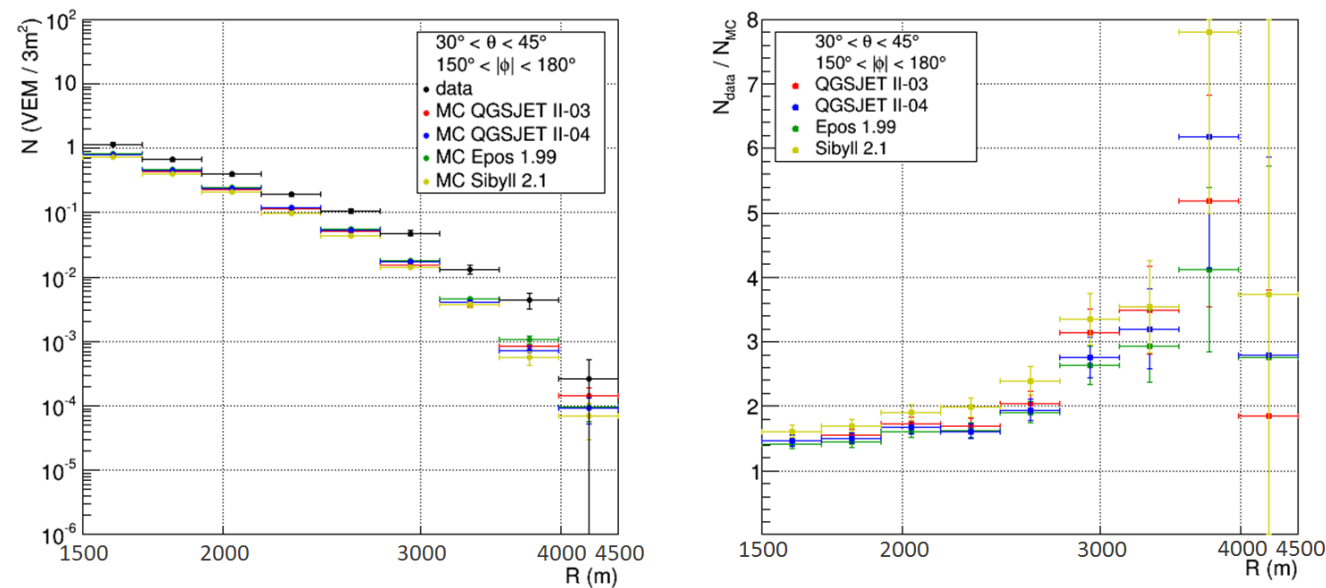

Figure 5: Same as figure 4 but for the MC with various hadronic models. (left) Lateral distributions of the average signal size assuming the histograms follow the Poisson distribution. The black, red, blue, green and yellow represent data, QGSJET II-03, QGSJET II-04, Epos 1.99 and Sibyll 2.1, respectively. (right) The average ratio of the data to the MC. The color corresponds to MC hadronic models described in the left figure.

and the ratio of the signal size of the data to that of the MC. On the high muon purity condition $\left(30^{\circ}<\theta<45^{\circ}, 150^{\circ}<|\phi|<180^{\circ}, 2000 \mathrm{~m}<R<4000 \mathrm{~m}\right.$, magenta filled circle in figure 6), the muon purity and the ratio of the data to the $\mathrm{MC}$ are $65 \%$ and $1.88 \pm 0.08$ (stat.) \pm 0.43 (syst.), respectively. In the case of the low muon purity condition $\left(\theta<30^{\circ},|\phi|<30^{\circ}, 2000 \mathrm{~m}<R<4000\right.$ $\mathrm{m}$, black open circle in figure 6 , they are calculated to be $28 \%$ and $1.30 \pm 0.06$ (stat.) \pm 0.31 (syst.), respectively. This figure shows larger difference in the signal size between the data and the MC at the higher muon purity. These results imply that part of the discrepancy between the data and the MC is explained by the muon excess in the data.

\section{Summary}

We studied muons in the air shower induced by UHECR with the TA SD to test hadronic interaction models of the air shower MC. The air shower events and the locations of SDs were divided on $\theta, \phi$ and $R$ conditions to search for the high muon purity analysis condition. We compared air shower signals of the data with that of the MC on the high muon purity condition $\left(10^{18.8}\right.$ $\left.\mathrm{eV}<E<10^{19.2} \mathrm{eV}, 30^{\circ}<\theta<45^{\circ}, 150^{\circ}<|\phi|<180^{\circ}, 2000 \mathrm{~m}<R<4000 \mathrm{~m}\right)$. On that condition, the muon purity expected from the $\mathrm{MC}$ is $\sim 65 \%$, and the typical ratios of the signal size of the data to that of the MC are $1.72 \pm 0.10$ (stat.) \pm 0.40 (syst.) at $1910 \mathrm{~m}<R<2160 \mathrm{~m}$ and $3.14 \pm 0.36$ (stat.) \pm 0.72 (syst.) at $2760 \mathrm{~m}<R<3120 \mathrm{~m}$. The lateral distribution of the data falls down slower than the MC on the high muon purity condition, causing the larger ratio of the data to the MC at the larger $R$ value. The difference in the signal size between the data and the MC is larger at the higher muon purity, which implies that part of the discrepancy between the data and the MC is explained by the muon excess in the data. 

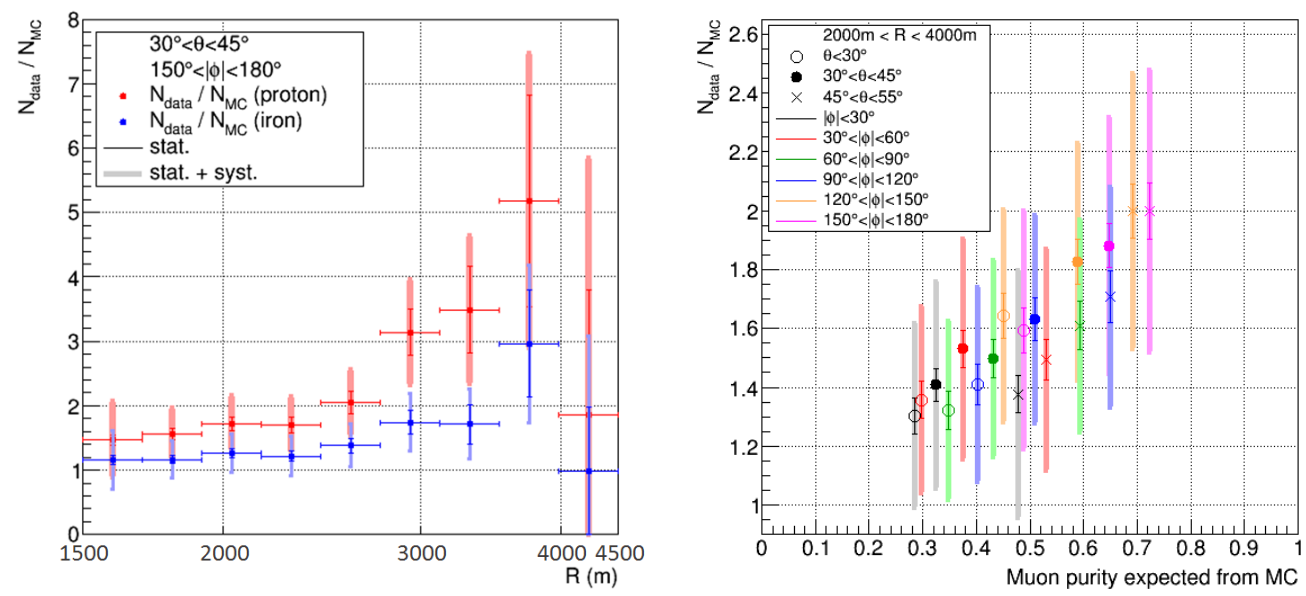

Figure 6: (left) The ratios of the signal size of the data to the MC for proton and iron. The vertical thin error bars and shaded thick error bars represent the statistical errors and the quadratic sum of statistical and systematic errors, respectively. The red and blue points represent the ratio of the data to the MC for proton and that for iron, respectively. (right) The correlation between the muon purity and the ratio of the signal size of the data to the MC with QGSJET II-03 model for $2000 \mathrm{~m}<R<4000 \mathrm{~m}$. The black, red, green, blue, orange and magenta represent $|\phi|<30^{\circ}, 30^{\circ}<|\phi|<60^{\circ}, 60^{\circ}<|\phi|<90^{\circ}, 90^{\circ}<|\phi|<120^{\circ}$, $120^{\circ}<|\phi|<150^{\circ}$ and $150^{\circ}<|\phi|<180^{\circ}$, respectively. The open circle, filled circle and cross represent $\theta<30^{\circ}, 30^{\circ}<\theta<45^{\circ}$ and $45^{\circ}<\theta<55^{\circ}$, respectively. The vertical thin error bars and shaded thick error bars represent the statistical errors and quadratic sum of statistical and systematic errors, respectively.

The feature obtained in this work that the signal of the data is larger than that of the MC is qualitatively consistent with the muon excess detected in researches of the Pierre Auger Observatory. In addition to the Auger analysis, where the $R$ dependence of muons is not studied, we confirmed the lateral distribution of the MC did not reproduce the data under the conditions on $\theta, \phi$ and $R$.

Acknowledgement : The Telescope Array experiment is supported by the Japan Society for the Promotion of Science through Grants-in-Aid for Scientific Research on Specially Promoted Research (21000002) "Extreme Phenomena in the Universe Explored by Highest Energy Cosmic Rays" and for Scientific Research (19104006), and the Inter-University Research Program of the Institute for Cosmic Ray Research; by the U.S. National Science Foundation awards PHY-0307098, PHY-0601915, PHY-0649681, PHY-0703893, PHY-0758342, PHY-0848320, PHY-1069280, PHY1069286, PHY-1404495 and PHY-1404502; by the National Research Foundation of Korea (2015R1A2A1A01006870，2015R1A2A1A15055344， 2016R1A5A1013277， 2007-0093860, 2016R1A2B4014967); by the Russian Academy of Sciences, RFBR grant 16-02-00962a (INR), IISN project No. 4.4502.13, and Belgian Science Policy under IUAP VII/37 (ULB). The foundations of Dr. Ezekiel R. and Edna Wattis Dumke, Willard L. Eccles, and George S. and Dolores Doré Eccles all helped with generous donations. The State of Utah supported the project through its Economic Development Board, and the University of Utah through the Office of the Vice President for Research. The experimental site became available through the cooperation of the Utah 
School and Institutional Trust Lands Administration (SITLA), U.S. Bureau of Land Management (BLM), and the U.S. Air Force. We appreciate the assistance of the State of Utah and Fillmore offices of the BLM in crafting the Plan of Development for the site. We also wish to thank the people and the officials of Millard County, Utah for their steadfast and warm support. We gratefully acknowledge the contributions from the technical staffs of our home institutions. An allocation of computer time from the Center for High Performance Computing at the University of Utah is gratefully acknowledged.

\section{References}

[1] M. Fukushima et al., Telescope Array project for extremely high energy cosmic rays, Prog. Theor. Phys. Suppl. 151, 206 (2003).

[2] T. Stroman et al., Telescope Array measurement of UHECR composition from stereoscopic fluorescence detection, Proc. 34th ICRC, 361 (2015).

[3] T. Fujii et al., Energy Spectrum and Mass Composition of Ultra-High Energy Cosmic Rays Measured with the Telescope Array Fluorescence Detector Using a Monocular Analysis, Proc. 34th ICRC, 320 (2015).

[4] A. Aab et al., Muons in air showers at the Pierre Auger Observatory: Mean number in highly inclined events, Phys. Rev. D 91, 032003 (2015).

[5] S. Ostapchenko, QGSJET-II: results for extensive air showers, Nucl. Phys. B, Proc. Suppl. 151, 147 (2006).

[6] S. Ostapchenko, Monte Carlo treatment of hadronic interactions in enhanced Pomeron scheme: QGSJET-II model, Phys. Rev. D 83, 014018 (2011).

[7] K. Werner, F. M. Liu, and T. Pierog, Parton ladder splitting and the rapidity dependence of transverse momentum spectra in deuteron-gold collisions at the BNL Relativistic Heavy Ion Collider, Phys. Rev. C., 74, 044902 (2006).

[8] A. Aab et al., Testing Hadronic Interactions at Ultrahigh Energies with Air Showers Measured by the Pierre Auger Observatory, Phys. Rev. Lett., 117, 192001 (2016).

[9] T. Abu-zayyad et al., The surface detector array of the Telescope Array experiment, Nucl. Instrum. Methods. A 689, 87 (2012).

[10] T. Abu-Zayyad et al., The Cosmic Ray Energy Spectrum Observed with the Surface Detector of the Telescope Array Experiment, ApJ 768, L1 (2013).

[11] R.U. Abbasi et al., Study of Ultra-High Energy Cosmic Ray composition using Telescope Array's Middle Drum detector and surface array in hybrid mode, Astropart. Phys. 64, 49 (2015).

[12] D. Ikeda et al., Energy spectrum and mass composition of ultra-high energy cosmic rays measured by the hybrid technique in Telescope Array, Proc. 34th ICRC, 362 (2015).

[13] T. Pierog and K. Werner, EPOS Model and Ultra High Energy Cosmic Rays, Nucl. Phys. Proc. Suppl. 196, 102 (2009).

[14] E. J. Ahn et al., Cosmic ray interaction event generator SIBYLL 2.1, Phys. Rev. D 80, 094003 (2009).

[15] T. Abu-Zayyad et al., Energy spectrum of ultra-high energy cosmic rays observed with the Telescope Array using a hybrid technique, Astropart. Phys. 61, 93 (2015). 